\title{
Use of solvent extraction to remediate soils contaminated with hydrocarbons
}

\author{
Aurora Silva $^{a}$, Cristina Delerue-Matos ${ }^{a}, *$ A. Fiúza ${ }^{b}$ \\ a REQUIMTE, Instituto Superior de Engenharia do Porto, R. António Bernardino de Almeida, \\ 431 4200-072 Porto, Portugal

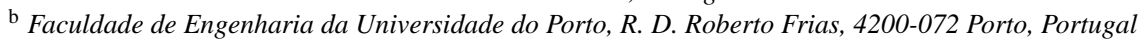 \\ Received 21 January 2005; received in revised form 9 May 2005; accepted 12 May 2005 \\ Available online 11 July 2005
}

\begin{abstract}
The main objective of this research is to exploit the possibility of using an ex situ solvent extraction technique for the remediation of soils contaminated with semi-volatile petroleum hydrocarbons. The composition of the organic phase was chosen in order to form a single phase mixture with an aqueous phase and simultaneously not being disturbed (forming stable emulsions) by the soil particles hauling the contaminants. It should also permit a regeneration of the organic solvent phase.

As first, we studied the miscibility domain of the chosen ternary systems constituted by ethyl acetate-acetone-water. This system proved to satisfy the previous requirements allowing for the formation of a single liquid phase mixture within a large spectrum of compositions, and also allowing for an intimate contact with the soil.

Contaminants in the diesel range within different functional groups were selected: xylene, naphthalene and hexadecane. The analytical control was done by gas chromatography with FID detector.

The kinetics of the extractions proved to be fast, leading to equilibrium after $10 \mathrm{~min}$. The effect of the solid-liquid ratio on the extraction efficiency was studied. Lower S/L ratios $(1: 8$, w/v) proved to be more efficient, reaching recoveries in the order of $95 \%$. The option of extraction in multiple contacts did not improve the recovery in relation to a single contact. The solvent can be regenerated by distillation with a loss around $10 \%$. The contaminants are not evaporated and they remain in the non-volatile phase.

The global results show that the ex situ solvent extraction is technically a feasible option for the remediation of semi-volatile aromatic, polyaromatic and linear hydrocarbons.
\end{abstract}

(C) 2005 Elsevier B.V. All rights reserved.

Keywords: Solvent extraction; Hydrocarbons; Soil

\section{Introduction}

Solvent extraction is a promising technology for soil remediation. It is an ex situ separation and concentration process in which a non-aqueous liquid is used to remove organic contaminants. The effectiveness of the extraction of pollutants from the soil depends on the realization of an intimate contact between the soil and the mixture of solvents. Consequently, some solvent extraction processes use water miscible

\footnotetext{
* Corresponding author. Tel.: +351 22 8340500; fax: +351 228321159 .

E-mail address: cmm@isep.ipp.pt (C. Delerue-Matos).
}

solvents or else the soils must be dried before applying the solvent extraction technique [1].

Although variants to this technology have been thoroughly described [2-5] some authors refer to complex mixtures of solvents, not always easy to find at lower costs, and some of them with a potential negative environmental impact.

The solvent extraction method have been accepted as an alternative for the remediation of soils contaminated with polychlorinated biphenyls (PCBs), when integrated with a complementary dechlorination technology [6]. Recently, the results of a study to assess the suitability of the solvent extraction soil remediation process have been tested for Aro- 
clor 1016, as an example of PCB in which solvent extraction is combined with solids agglomeration. A two-stage extraction process, with a series of three wash steps incorporated into the solid-liquid separation operation produced a treated soil with recovery rates of Aroclor 1016 above $94 \%$ [7].

The remediation of soils contaminated with polycyclic aromatic hydrocarbons (PAHs) has also been researched and several solvents are reported: ethanol, 2-propanol, acetone, 1-pentanol and water. The extraction data for several PAHs compounds indicate that while 1-pentanol was more effective in removing naphthalene and less effective with four-ring PAHs, removals were generally comparable for ethanol, 2propanol, acetone and 1-pentanol. Since water was present in the soil moisture, it was considered as a component of the extracting solvent. From the various mixtures of solvents, the ternary one composed by 5\% 1-pentanol, $10 \%$ water and $85 \%$ ethanol was selected for further optimization of the extraction process, using $1 \mathrm{~h}$ of contact time between soil and solvent mixture. A three-stage crosscurrent solvent washing train was capable of removing more than $95 \%$ of the PAH contamination from highly contaminated field soils [8].

The use of hot water in a subcritical state, at approximated $250{ }^{\circ} \mathrm{C}$, was described reporting removals of PAH's between 79 and $99 \%$ depending on the molecular weight of the contaminant, for an optimized extraction time of $2 \mathrm{~h}$ [9].

Solvent washing was evaluated as a method to remove pentachlorophenol (PCP) from aged field soils contaminated with wood treating wastes. The $50 \%$ ethanol solution proved to be as effective as more concentrated solutions of ethanol in removing PCP from field soils in batch extraction tests. Maximum observed PCP removal from field soils occurred within an hour of contact time between soil and solvent [10]. A crosscurrent soil washing procedure was developed and at least two wash stages with $50 \%$ ethanol were needed to remove more than $77 \%$ of the PCP. Ethanol recoveries were greater than $92 \%$ when the crosscurrent washing processes where followed by water rinse stages. In three-stage solvent washing process counter current washing removed nearly three times more PCP per volume of solvent than a similar crosscurrent solvent washing process. This study was also conducted to determine whether soil solvent washing would also be considered as a cleanup alternative for petroleum hydrocarbons [11].

The mixture acetone-ethyl acetate-water became interesting because it consists of environmental friendly solvents, and specially because for certain compositions, they form a single phase mixture [1], which allows a more efficient contact with the soil, allowing the application in wet soils and sediments without a previous drying operation. To study the capacity of this mixture to remove petroleum compounds from the soil, we selected three compounds belonging to the diesel range [12]: xylene, naphthalene and hexadecane. The selection criterion was based on the choice of hydrocarbons with different structures, namely one aromatic (xylene), one polyaromatic (naphthalene) and one linear (hexadecane).

\section{Materials and methods}

\subsection{Reagents}

The reagents $n$-pentane, xylene, naphthalene, potassium dichromate, ammonium iron sulphate were all p.a. grade and supplied by Merck. The hexadecane was supplied by Sigma-Aldrich and also at p.a. grade. Acetone and ethyl acetate were supplied by Merck at pure grade. The water used was deionised and had a conductivity less than $0.1 \mu \mathrm{S} / \mathrm{cm}$.

\subsection{Analytical conditions}

The analytical method used in the quantification of the contaminants was based on the Texas Natural Resource Conservation Commission (TNRCC) Method 1005, which is a generic procedure for the determination of hydrocarbons in soil samples using a chromatographic procedure [13].

The gas chromatograph used was a Chrompack CP9000, equipped with a FID detector and a split injector. The separations were performed using a CP-SIL $8 \mathrm{CB}$ Chrompack fused silica capillary column $(25 \mathrm{~m} \times 0.25 \mathrm{~mm}$ i.d. $\times 0.12 \mu \mathrm{m}$ of film thickness). The chromatographic conditions were the following: an initial oven temperature of $50{ }^{\circ} \mathrm{C}$ for $3 \mathrm{~min}$, followed by an increase in the temperature of $25^{\circ} \mathrm{C} / \mathrm{min}$ until $310^{\circ} \mathrm{C}$. The injector and the detector temperatures were kept at 285 and $325^{\circ} \mathrm{C}$, respectively. The gases used in chromatographic analysis were hydrogen (34 $\mathrm{mL} / \mathrm{min})$, air $(300 \mathrm{~mL} / \mathrm{min})$ and nitrogen $(20 \mathrm{~mL} / \mathrm{min})$, the last one used as carrier. Quantification was based one the area count obtained for the peaks of the extracts and they were compared to standards used in the calibration curve. The solvent used in the analysis was the $n$-pentane chosen because it does not interfere in the analytical process. The injection volume used was $1 \mu \mathrm{L}$.

\subsection{Ternary diagram}

For the experimental determination of the ternary diagram acetone-ethyl acetate-water, different volumes of acetone-water and acetone-ethyl acetate were taken, after which an accurate volume of the third compound was added until the occurrence of immiscibility was observed. The experiment was done at room temperature.

\subsection{Soil preparation}

The soil used was collected at an agricultural area in north of Portugal, without previous history of industrial activities, or suspicion of previous hydrocarbons contamination. It was put in an oven, at $50{ }^{\circ} \mathrm{C}$ for $24 \mathrm{~h}$, to equalize the moisture 
content and after screening ( 10 mesh) to exclude the fractions larger than $2 \mathrm{~mm}$.

\subsection{Soil characterization methods}

The main properties assessed in the soil were the $\mathrm{pH}$, the bulk density, the moisture content, the organic carbon content and the size distribution.

The $\mathrm{pH}$ was measured directly in the supernatant with a combined glass electrode [14]. The bulk density was evaluated by using the relation mass and volume $[14,15]$, and the moisture content was measured by mass difference after $24 \mathrm{~h}$ drying at $105^{\circ} \mathrm{C}$ [14]. The organic carbon content was determined by wet oxidation at $200^{\circ} \mathrm{C}$ in the presence of potassium dichromate [16]; the excess of dichromate was evaluated by titration with ammonium iron sulphate $[14,15]$.

\subsection{Soil contamination}

Approximately $2.5 \mathrm{~kg}$ of soil was contaminated with $7.04200 \mathrm{~g}$ of xylene, $6.11336 \mathrm{~g}$ of naphthalene and $7.34473 \mathrm{~g}$ of hexadecane. Because naphthalene is solid at room temperature, this contaminant was previously dissolved in trymethylpentane before it was spiked in the soil. After the contamination, the soil remained in a closed box for 2 weeks to allow the dispersion and sorption of the contaminant in the soil matrix.

\subsection{Solid-liquid extraction}

To evaluate the capacity of the acetone-ethyl acetatewater mixture to remove the contaminants from the soil, the following procedure was adopted: to a rigorous mass of contaminated soil, around $25 \mathrm{~g}$, a know volume of solvent mixture was added. The contact between the phases was promoted by magnetic agitation and the separation was done by vacuum filtration. Samples of soil and samples of the extract mixture were collected for later gas chromatographic analysis.

\subsection{Distillation}

The solvent regeneration experiments were performed in a vigreux column with $30 \mathrm{~cm}$ of length and $10 \mathrm{~cm}$ of diameter; $100 \mathrm{~mL}$ of mixture was used in each experiment.

\section{Results and discussion}

\subsection{Soil characterization}

Detailed physical and chemical characteristics of the soil sample used for this study were determined and the results are summarised in Table 1.
Table 1

Soil characteristics

\begin{tabular}{lc}
\hline Parameter & Value \\
\hline $\mathrm{pH}$ & 5.84 \\
Moisture (\%) & 7.79 \\
Bulk density (g/L) & 950.2 \\
Organic carbon (\%) & 1.314 \\
Clays (\%) & 6.89 \\
Silts (\%) & 63.41 \\
Sand (\%) & 29.70 \\
\hline
\end{tabular}

\subsection{Analytical}

For each one of the studied pollutants, a calibration curve was obtained using a large frame of standards solutions to include all the possible working areas. All the determinations were made in triplicate. A chromatogram of a standard in the optimized conditions is presented, as example, in Fig. 1.

A standard calibration method was used to quantify the pollutant in soil, using nine standard solutions, with concentrations in the $5-800 \mathrm{mg} / \mathrm{L}$ range; a linear response was obtained. The linear correlation parameters [17] slope, intercept, detection limit and the correlation coefficient $\left(R^{2}\right)$ are presented in Table 2.

\subsection{Ternary diagram}

The experimental data conducive to the isothermal ternary phase diagram of ethyl acetate, acetone and water are shown in Table 3, where all values are expressed as mass fractions. A non-miscibility region is present, owing to the partial mutual miscibility of ethyl acetate and water [1]. These results agree well with the diagram described by Nardella et al. The miscibility gap is above the points represented in Fig. 2.

From the ternary phase diagram several mixtures were selected from A to $\mathrm{H}$ to evaluate the influence of composition of the extract mixture in the removal of pollutants from contaminated soil.

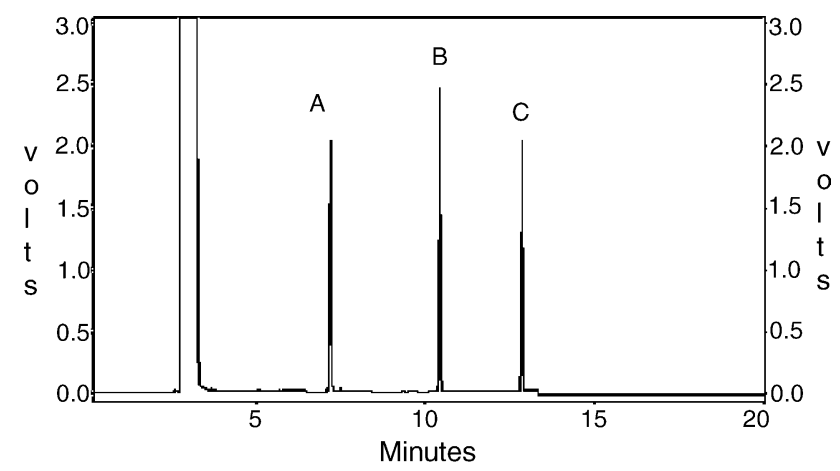

Fig. 1. Chromatogram of standard approximately $200 \mathrm{mg} / \mathrm{L}$ in each pollutant: (A) xylene, (B) naphthalene and (C) hexadecane. 
Table 2

Calibration parameters

\begin{tabular}{lllll}
\hline Pollutant & Slope & Intercept & Detection limit $(\mathrm{mg} / \mathrm{L})$ & $R^{2}$ \\
\hline Xylene & $16546 \pm 128.56$ & $84369 \pm 42025.21$ & 1.31 & 0.9993 \\
Naphthalene & $18331 \pm 95.20$ & $29867 \pm 31211.28$ & 4.39 & 0.9998 \\
Hexadecane & $17849 \pm 125.88$ & $20401 \pm 37304.84$ & 1.31 & 0.9997 \\
\hline
\end{tabular}

Table 3

Data obtained for the ternary phase diagram $(\%)$

\begin{tabular}{lll}
\hline Ethyl acetate & Acetone & Water \\
\hline 0.394 & 0.356 & 0.250 \\
0.416 & 0.358 & 0.226 \\
0.439 & 0.356 & 0.205 \\
0.508 & 0.339 & 0.153 \\
0.130 & 0.120 & 0.750 \\
0.150 & 0.270 & 0.570 \\
0.210 & 0.310 & 0.480 \\
0.250 & 0.330 & 0.410 \\
0.710 & 0.190 & 0.100 \\
0.620 & 0.270 & 0.100 \\
0.238 & 0.336 & 0.426 \\
0.322 & 0.356 & 0.322 \\
0.384 & 0.361 & 0.254 \\
0.409 & 0.362 & 0.229 \\
0.431 & 0.361 & 0.208 \\
0.123 & 0.120 & 0.758 \\
0.155 & 0.272 & 0.573 \\
0.209 & 0.306 & 0.484 \\
0.252 & 0.330 & 0.418 \\
0.544 & 0.335 & 0.121 \\
0.602 & 0.302 & 0.096 \\
0.555 & 0.332 & 0.112 \\
\hline
\end{tabular}

\subsection{Solvent extraction}

Batch extraction experiences were conducted with various ethyl acetate-acetone-water mixtures using a volume of $50 \mathrm{~mL}$ for each mixture and a contact time of $15 \mathrm{~min}$. The results obtained are shown in Table 4.

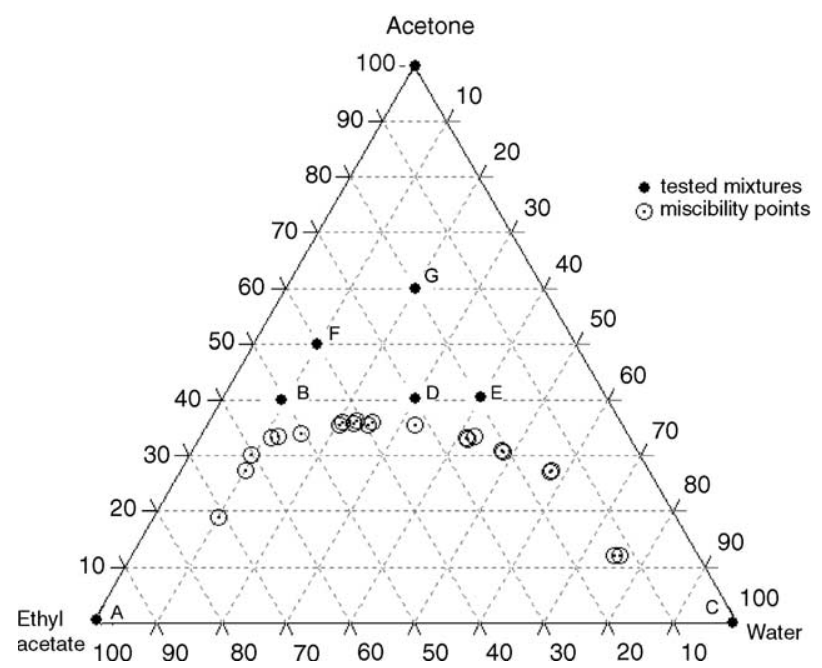

Fig. 2. Experimental ternary phase diagram ethyl acetate-acetone-water and selected mixtures.
Table 4

Removal values obtained for the studied mixtures

\begin{tabular}{|c|c|c|c|c|c|c|}
\hline \multicolumn{4}{|c|}{ Composition (\%) } & \multicolumn{3}{|c|}{ Removal (\%) } \\
\hline & Water & Acetone & Ethyl acetate & Xylene & Naphthalene & Hexadecane \\
\hline A & 0 & 0 & 100 & 86.66 & 86.65 & 87.36 \\
\hline $\mathrm{B}$ & 10 & 40 & 50 & 83.37 & 84.24 & 83.78 \\
\hline $\mathrm{C}$ & 100 & 0 & 0 & 26.84 & 15.87 & - \\
\hline $\mathrm{D}$ & 30 & 40 & 30 & 64.11 & 65.81 & 64.52 \\
\hline $\mathrm{E}$ & 40 & 40 & 20 & 79.17 & 71.11 & 57.73 \\
\hline $\mathrm{F}$ & 10 & 50 & 40 & 75.21 & 73.97 & 77.06 \\
\hline $\mathrm{G}$ & 20 & 60 & 20 & 74.45 & 70.53 & 74.30 \\
\hline $\mathrm{H}$ & 0 & 100 & 0 & 78.21 & 77.62 & 79.04 \\
\hline
\end{tabular}

By observing these results it can be concluded that ethyl acetate is the compound that has a higher contribution to the removal efficiency, in contrast to the water. Multivariable linear regressions confirmed this conclusion. The choice of a mixture of $50 \%$ ethyl acetate, $40 \%$ of acetone and $10 \%$ of water was made because it was necessary to assure the existence of a single liquid phase, in order to promote an efficient contact between solid and liquid.

\subsection{Kinetics of the hydrocarbons removal}

The removal of the contaminants from the soil was studied as a function of time using a mixture composition of $50 \%$ ethyl acetate, $40 \%$ acetone and $10 \%$ water. The kinetics data are presented in Fig. 3a-c.

The results show that the extraction process rapidly reaches equilibrium, at a time of approximately $10 \mathrm{~min}$, for

Table 5

Variation of the removal rate, with the increase of the extraction mixture volume

\begin{tabular}{llll}
\hline & \multicolumn{3}{l}{ Removal rate $(\%)$} \\
\cline { 2 - 4 } Solid-liquid ratio & Xylene & Naphthalene & Hexadecane \\
\hline $1: 1$ & 66.83 & 67.94 & 68.78 \\
$1: 2$ & 86.24 & 86.33 & 87.33 \\
$1: 3$ & 90.63 & 90.29 & 89.40 \\
$1: 4$ & 94.04 & 93.60 & 93.10 \\
$1: 8$ & 94.81 & 94.96 & 95.16 \\
\hline
\end{tabular}

Table 6

Removal rates obtained in soil and sand

\begin{tabular}{lll}
\hline & \multicolumn{2}{l}{ Removal $(\%)$} \\
\cline { 2 - 3 } & Sand & Soil \\
\hline Naphthalene & 95.96 & 84.49 \\
Hexadecane & 96.26 & 84.98
\end{tabular}



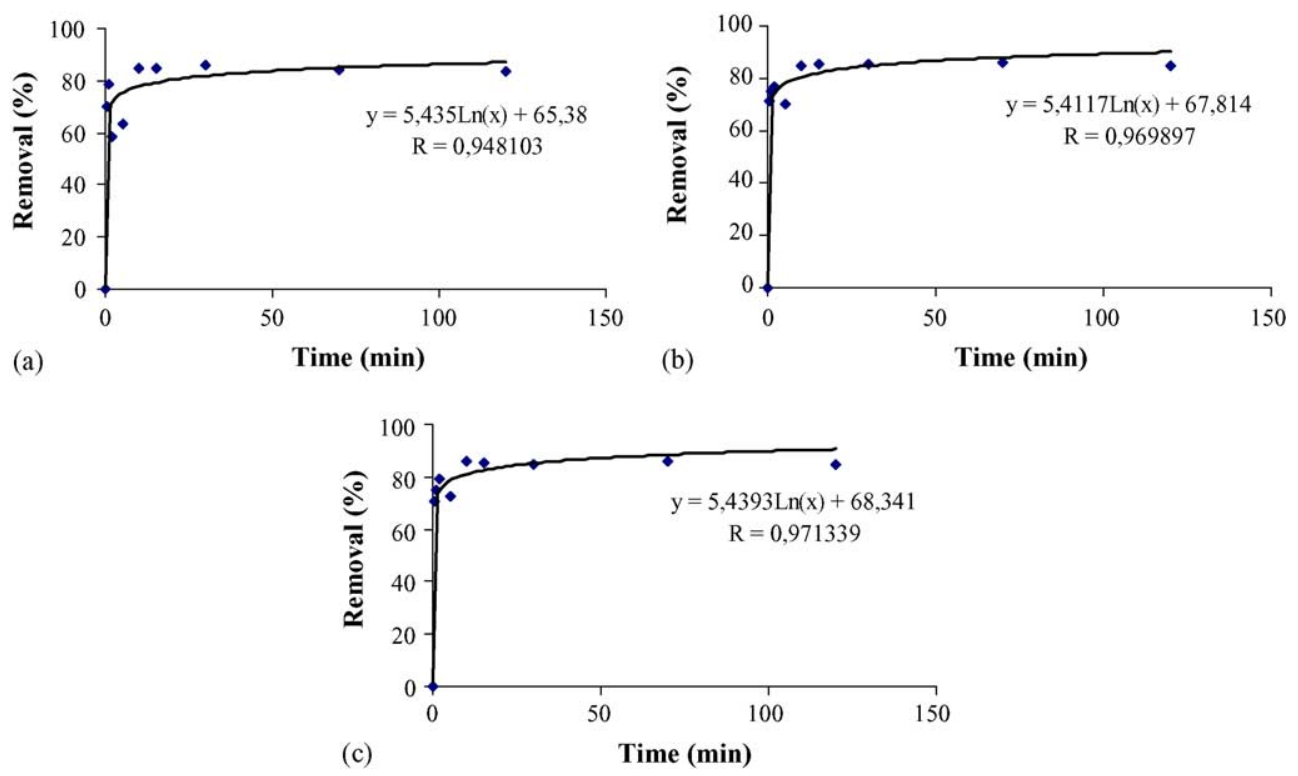

Fig. 3. Extraction kinetics for (a) xylene, (b) naphthalene and (c) hexadecane.

Table 7

Distillation mass balance

\begin{tabular}{|c|c|c|c|c|c|}
\hline & \multirow[t]{2}{*}{ Initial mass (mg) } & \multicolumn{2}{|c|}{ Mass in the organic phase (mg) } & \multicolumn{2}{|c|}{ Mass in the aqueous phase (mg) } \\
\hline & & First experience & Replicate & First experience & Replicate \\
\hline Xylene & 2.84 & 0.17 & 0.52 & 2.27 & 1.88 \\
\hline Naphthalene & 22.98 & 0.31 & 0.18 & 20.21 & 20.64 \\
\hline Hexadecane & 28.84 & 0.54 & 0.33 & 24.44 & 24.17 \\
\hline
\end{tabular}

each hydrocarbon; after this period no considerable changes in the removal rates were observed.

\subsection{Soil/solvent ratio}

Following the kinetics studies, and using the optimized contact time of $15 \mathrm{~min}$, the solid-liquid ratio was considered. For a constant mass of soil ( $25 \mathrm{~g}$ ), different volumes of the extracting mixture were used: $25,50,75,100$ and $200 \mathrm{~mL}$ corresponding to solid-liquid ratios of 1:1, 1:2, 1:3, 1:4 and 1:8(w/v). The results are shown in Table 5.

These results indicate that the three pollutants have a similar behaviour. The recovery efficiency increases when the liquid/soil ratio increases. For all contaminants, the removal rate improved in average $40 \%$ when the liquid phase was eight times higher than the solid one, conducting to removal level near $95 \%$. Increase in the liquid phase provides a higher mass transfer from the soil under study. This is a result of two main factors: improve of the contact between phases and the increases on concentrations gradient. From the results indicated in Table 5, it seems that the three contaminants present similar physico-chemical behaviour at the experimental condition under study.

The option of operating with successive contact stages of the solvent mixture was also considered, but the levels of remediation obtained were not significantly different from the results achieved when the same volume of the extracting mixture was used in a single contact stage.

To evaluate the influence of organic carbon content, an experience was performed with soil and sand in similar conditions. The results obtained (Table 6) show that the removal rates in sand, a matrix whit low organic carbon content $(0.017 \%)$, are $13 \%$ higher than the removal rates in soil. Xylene does not sorbs enough in the sand to be measured so was not considered in this experience.

\subsection{Solvent regeneration}

After solid-liquid separation the single phase spent liquid mixture used in each extraction was used to perform the regeneration experiments. Distillation was selected as the regeneration technique, in the experimental conditions described in 2.8. Acetone and ethyl acetate distil together at $65{ }^{\circ} \mathrm{C}$ of vapour temperature. The results obtained in the regeneration experiments are summarised in Table 7.

The mass balance indicates losses of approximately $10 \%$ in the distillation process. These results indicate that the distillation is an effective technique to regenerate the spent solvent mixture. The aqueous phase after distillation can by remit to a water treatment station. 


\section{Conclusions}

These studies show that the environmentally friendly mixture of ethyl acetate, acetone and water is effective in removing hydrocarbon compounds from the soils. In this mixture, the compound that contributes more to the remediation efficiency is the ethyl acetate.

The extraction kinetics is fast reaching equilibrium after $10 \mathrm{~min}$, corresponding to a hydrocarbon removal of $85 \%$.

A solid-liquid ratio of $1: 2$ is sufficient to achieve an acceptable remediation level, but when that ratio is increased to $1: 8$ the removal further increases $8 \%$ in average. The multiple contact options were considered but no significant difference in the results was observed. In future applications, it will be necessary to adjust these two parameters to optimize the extraction efficiency and the target concentration.

As it is expected, carbon content is an important parameter in the extraction efficiency.

Distillation proves to be an applicable method to perform the solvent regeneration.

\section{References}

[1] A. Nardella, F. Massetti, R. Tomaciello, Clean up of polluted wet soils by solvent extraction, Environ. Prog. 18 (4) (1999) 243-249.

[2] U.S. Environmental Protection Agency, Soil Washing Treatment, U.S.EPA Office of Research and Development, Risk Reduction Engineering Laboratory, EPA/540/2-90/017, Cincinnati, OH, 1990.

[3] U.S. Environmental Protection Agency, Solvent Extraction Treatment, U.S.EPA Office of Emergency Response, EPA/540/2-90/013, Washington, DC, 1990.
[5] W.C. Anderson, Solvent/Chemical Extraction Innovative Site Remediation Technology, vol. 5, American Academy of Environmental Engineers, Annapolis, MD, 1995.

[6] R.D. Curry, T. Clevenger, O. Stancu-Ciolac, W.H. Miller, H. Farmer, B.J. Mincher, S. Kapila, Decontamination of soil contaminated with Aroclor 1260 using a solvent extraction process and $\gamma$-ray radiolysis, J. Adv. Oxid. Technol. 3 (1998) 55-62.

[7] S.A. Majid, B.D. Sparks, Removal of Arocol 1016 from contaminated soil by solvent extraction soil agglomeration process, J. Environ. Eng. Sci. 1 (2002) 59-64.

[8] A.P. Khodadoust, R. Bagchi, M.T. Suidan, R.C. Brenner, N.G. Sellers, Removal of PAHs from highly contaminated soils found at prior manufactured gas operations, J. Hazard. Mater. B 80 (2000) 159-174.

[9] A.A. Dadkhah, A. Akgerman, Hot water extraction with in situ oxidation: PAHs removal from soil, J. Hazard. Mater. B 93 (2002) 307-320.

[10] A.P. Khodadoust, M.T. Suidam, C.M. Acheson, R.C. Brenner, Solvent extraction of pentachlorophenol from contaminated soils using water-ethanol mixtures, Chemosphere 38 (11) (1999) 2681-2693.

[11] A.P. Khodadoust, M.T. Suidam, C.M. Acheson, R.C. Brenner, Remediation of soils contaminated with wood preserving wastes: crosscurrent and countercurrent solvent washing, J. Hazard. Mater. B 64 (1999) 167-179.

[12] W. Weisman, Analysis of Petroleum Hydrocarbons in Environmental Media, Air Force Laboratory Operational Toxicology, 1998.

[13] TNRCC-Method 1005, Total Petroleum Hydrocarbons, Texas Natural Resource Conservation Commission, 2001.

[14] M.R. Carter, Soil Sample and Methods of Analysis, Canadian Society of Soil Science, Lewis, 1993.

[15] K.H. Tan, Soil Sampling, Preparation and Analysis, Marcel Dekker, Inc., New York, 1996.

[16] C.G. Schreier, W.J. Walker, J. Burns, R. Wikenfeld, Total organic carbon as a screening method for petroleum hydrocarbons, Chemosphere 39 (3) (1998) 503-510.

[17] J.N. Miller, J.C. Miller, Statistics and Chemometrics for Analytical Chemistry, fourth ed., Prentice Hall, London, 2000. 